\title{
Dietary potassium regulates vascular calcification and arterial stiffness
}

\author{
Yong Sun, ${ }^{1}$ Chang Hyun Byon, ${ }^{1}$ Youfeng Yang, ${ }^{1}$ Wayne E. Bradley, ${ }^{2}$ Louis J. Dell'Italia, ${ }^{2}$ \\ Paul W. Sanders, ${ }^{2,3}$ Anupam Agarwal, ${ }^{2,3}$ Hui Wu, ${ }^{4}$ and Yabing Chen ${ }^{1,3}$ \\ 'Department of Pathology and 'Department of Medicine, University of Alabama at Birmingham, Birmingham, Alabama, \\ USA. ${ }^{3}$ Research Department, Veterans Affairs Birmingham Medical Center, Birmingham, Alabama, USA. ${ }^{4}$ Department of \\ Pediatric Dentistry, University of Alabama at Birmingham, Birmingham, Alabama, USA.
}

\begin{abstract}
Vascular calcification is a risk factor that predicts adverse cardiovascular complications of several diseases including atherosclerosis. Reduced dietary potassium intake has been linked to cardiovascular diseases such as hypertension and incidental stroke, although the underlying molecular mechanisms remain largely unknown. Using the ApoE-deficient mouse model, we demonstrated for the first time to our knowledge that reduced dietary potassium $(0.3 \%)$ promoted atherosclerotic vascular calcification and increased aortic stiffness, compared with normal $(0.7 \%)$ potassium-fed mice. In contrast, increased dietary potassium (2.1\%) attenuated vascular calcification and aortic stiffness. Mechanistically, reduction in the potassium concentration to the lower limit of the physiological range increased intracellular calcium, which activated a cAMP response element-binding protein (CREB) signal that subsequently enhanced autophagy and promoted vascular smooth muscle cell (VSMC) calcification. Inhibition of calcium signals and knockdown of either CREB or ATC7, an autophagy regulator, attenuated VSMC calcification induced by low potassium. Consistently, elevated autophagy and CREB signaling were demonstrated in the calcified arteries from low potassium diet-fed mice as well as aortic arteries exposed to low potassium ex vivo. These studies established a potentially novel causative role of dietary potassium intake in regulating atherosclerotic vascular calcification and stiffness, and uncovered mechanisms that offer opportunities to develop therapeutic strategies to control vascular disease.
\end{abstract}

Authorship note: Y. Sun and C.H. Byon contributed equally to this work.

Conflict of interest: The authors have declared that no conflict of interest exists.

Submitted: May 3, 2017

Accepted: August 24, 2017

Published: October 5, 2017

Reference information:

JCI Insight. 2017;2(19):e94920.

https://doi.org/10.1172/jci.

insight.94920.

\section{Introduction}

Vascular pathology comprises not only remodeling of resistance vessels, but also stiffening of compliance vessels. In the Cardiovascular Health Study, a prospective multicenter study of 4,476 men and women at 65 years of age or older, 79.9\% developed increased carotid artery intima and media thickness (1). As large arteries stiffen, left ventricular hypertrophy develops, which increases the rate of cardiovascular events (2, 3). Arterial stiffness has become an independent predictor of cardiovascular morbidity and mortality, representing an important health problem for the nation as a whole.

Emerging clinical evidence suggests the importance of vascular calcification in aortic stiffening (4-8), and vascular calcification is a risk factor that predicts adverse cardiovascular outcomes in several diseases, often with overlapping vascular complications such as atherosclerosis, diabetes, and chronic kidney disease (9). Previously considered a passive, unregulated, and degenerative process occurring in the arterial media, vascular calcification has now been demonstrated to be a highly regulated process of osteochondrogenic differentiation of vascular cells $(10,11)$. We and others have shown that osteogenic differentiation and calcification of vascular smooth muscle cells (VSMCs) contribute to vascular calcification in atherosclerosis (12-14). Calcifying VSMCs produce extracellular matrix proteins that further accelerate the development of arterial calcification $(15,16)$. Therefore, calcification of VSMCs results in alterations in the molecular and cellular functions of VSMCs, the main cell type that determines the vascular tone, and leads to disturbance of the vasculature and arterial stiffening (17).

Epidemiological studies have suggested a role for potassium in the clinical manifestations of cardiovascular disease. Inverse associations between serum potassium levels and risks of chronic kidney disease (18) and metabolic syndrome (19) have been documented in patients along with a higher propensity to develop cardiovascular calcification. In prospective cohort studies, low serum potassium levels are associated with significantly higher risks of cardiovascular mortality compared with patients who had serum potassium 
concentrations within the normal range $(20,21)$. Reduced potassium is associated with cardiovascular diseases such as hypertension and chronic heart failure, and appropriate dietary potassium intake improves those pathological conditions (22-24). Therefore, serum potassium levels are associated with cardiovascular health and disease; however, the mechanisms responsible for potassium-regulated cardiovascular complications are not fully understood.

At the cellular level, potassium has been shown to reduce VSMC proliferation (25) and inhibit VSMC migration in vitro (26). In animal models, elevation of extracellular potassium levels resulting from increased dietary potassium intake attenuated balloon injury-induced neointimal formation in rat carotid arteries, possibly through inhibition of proliferation and migration of VSMCs (27). In a population-based study, high levels of dietary potassium have been postulated to impair proliferation of VSMCs, and reduce monocyte adherence to vessel walls, thereby retarding the progression of atherosclerosis (28). Nonetheless, a direct effect of dietary potassium on VSMC osteogenic differentiation and the development of vascular calcification in atherosclerosis has not been established and characterized.

In this study, we have provided the first evidence to our knowledge that supports a causative role of dietary potassium in regulating osteogenic differentiation and calcification of VSMCs in vitro and vascular calcification and stiffness in atherosclerotic animals in vivo. Furthermore, we defined key mechanisms underlying low-potassium-induced VSMC calcification involving elevated intracellular calcium, activated cAMP response element-binding protein (CREB), and increased autophagy. These findings provide molecular insights into the regulation of vascular calcification and stiffness by potassium, which explain the impact of dietary potassium intake on cardiovascular complications, and uncover potentially new targets to develop strategies to prevent and treat vascular complications.

\section{Results}

Dietary potassium regulated vascular calcification and aortic stiffness in mice. The effect of dietary potassium on atherosclerotic vascular calcification was characterized in vivo using the high-fat-fed $A p o E^{-/-}$mouse model $(12,13)$, with dietary intake of standard $(0.7 \% \mathrm{wt} / \mathrm{wt})$, low $(0.3 \% \mathrm{wt} / \mathrm{wt})$, or high $(2.1 \% \mathrm{wt} / \mathrm{wt})$ potassium, as previously reported $(29,30)$. Mice fed the $0.3 \%$ potassium diet exhibited significant increases in vascular calcification, compared with mice fed the $0.7 \%$ potassium diet, whereas the $2.1 \%$ potassium diet markedly inhibited vascular calcification (Figure 1, A and B). The effects of dietary potassium on vascular calcification were demonstrated in aortic root sections by Alizarin red staining (Figure 1, A and B), as well as descending aortas by total calcium quantification (Figure 1C). It is worth noting that mice fed the $0.3 \%$ potassium diet had lower mean serum potassium levels $(3.70 \pm 0.21 \mathrm{mEq} / 1)$, while mice fed the $2.1 \%$ potassium diet had higher serum potassium levels $(4.73 \pm 0.15 \mathrm{mEq} / 1)$, compared with levels $(4.27 \pm 0.23$ $\mathrm{mEq} / 1)$ observed in mice fed the standard $(0.7 \%$ potassium) diet (Supplemental Table 1; supplemental material available online with this article; https://doi.org/10.1172/jci.insight.94920DS1).

In line with our observation of elevated calcium content in the descending aortas, echocardiographic analysis revealed that the $0.3 \%$ potassium diet induced a significant increase in mean pulse wave velocity (PWV) (Figure 1D), an indicator of aortic stiffness (31), suggesting that impaired aortic compliance is associated with low dietary potassium-induced vascular calcification. In contrast, compared with animals fed the $0.7 \%$ potassium diet, animals fed the $2.1 \%$ potassium diet exhibited inhibited vascular calcification and concurrently reduced PWV, supporting an important role of dietary potassium in regulating vascular calcification and stiffness.

Potassium regulated vascular calcification ex vivo. To determine if there was a direct effect of extracellular potassium level on calcification of the arteries and VSMCs in their natural milieu, we employed an ex vivo ring culture model that we and others have recently developed for histological and quantitative analysis of arterial calcification $(32,33)$. Based on normal physiological levels of serum potassium in adult C57BL/6 mice (34-36), we determined the effects of potassium at the lower $\left(3.7 \mathrm{mM}\right.$, low $\left.\mathrm{K}^{+}\right)$, middle $(5.4 \mathrm{mM}$, control), and higher $\left(6.0 \mathrm{mM}\right.$, high $\left.\mathrm{K}^{+}\right)$end of the physiological range on aortic calcification. Consistent with the in vivo results, we found that low potassium markedly enhanced vascular calcification in the aortic media, as demonstrated by Alizarin red staining (Figure 2A), while high potassium inhibited aortic calcification. Quantification of total calcium content demonstrated a significant increase in calcification in aortic rings cultured in medium containing $3.7 \mathrm{mM}$ potassium, which was inhibited by $6.0 \mathrm{mM}$ potassium (Figure $2 \mathrm{~B}$ ). These results demonstrated a direct effect of potassium on the calcification of the aortic media, supporting the role of low potassium in promoting VSMC calcification. 
A
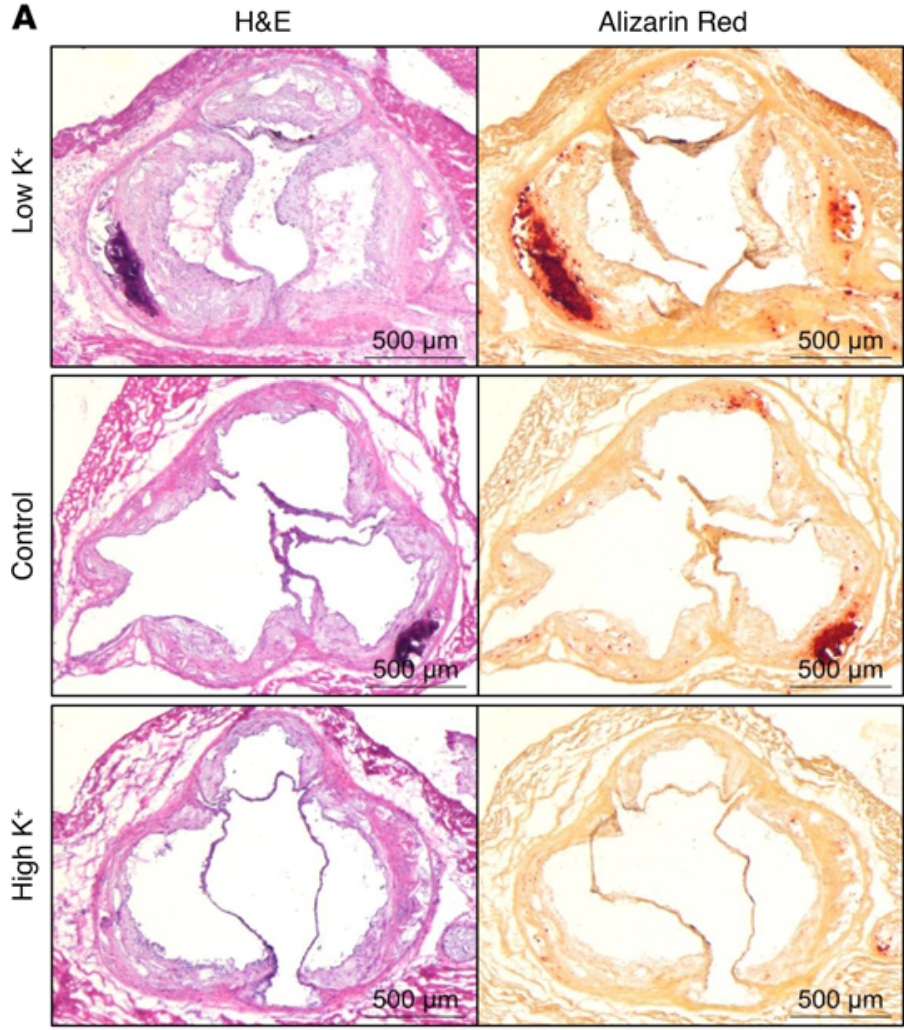

B
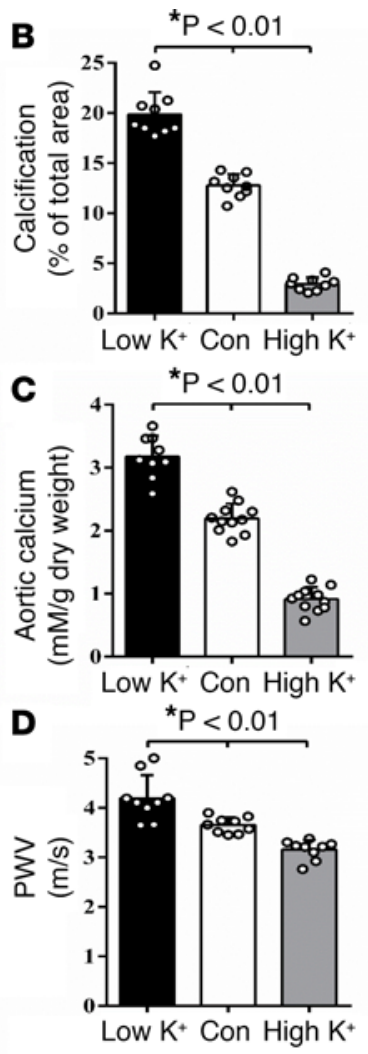

Figure 1. Dietary potassium regulated vascular calcification and aortic stiffness in mice. $A p o E^{-/-}$mice $(n=9 / g r o u p)$ were fed a high-fat diet containing normal potassium (Control), low potassium (Low K+) or high potassium (High $\mathrm{K}^{+}$) for 30 weeks. (A) Vascular calcification in aortic roots, determined by Alizarin red staining. Representative images of H\&E staining and Alizarin red staining in consecutive aortic root sections. Scale bars: $500 \mu \mathrm{m}$. (B) Quantification of calcification in the aortic root sections, measured using Image) software. Results presented are the percentage of positively stained areas in the total atherosclerotic lesion area of aortic roots. Bar values are means \pm SD. (C) Total calcium content in the descending aortas, quantified by the Arsenazo III method. Results shown are normalized by total protein amount. Bar values are means \pm SD. (D) Effects of dietary potassium on aortic stiffness. Pulse wave velocity (PWV), an indicator for aortic stiffness, determined by echocardiography at the end of the experiments. Bar values are means \pm SD. Statistical analysis was performed by 1-way ANOVA followed by a Student-Newman-Keuls test.

Lower physiological levels of potassium induced VSMC osteogenic differentiation and calcification. To determine the effects of potassium on VSMC calcification in cell culture, we first assessed the effects of extracellular potassium on VSMC viability using potassium at concentrations up to $108 \mathrm{mM}$, so as to identify a nontoxic level of potassium. Consistent with previous observations (37), we found that potassium, at concentrations lower than $54 \mathrm{mM}$, did not affect VSMC viability (Supplemental Figure 1). However, at concentrations from 3.7 to $4.7 \mathrm{mM}$, potassium markedly enhanced VSMC calcification compared with its concentration at $5.4 \mathrm{mM}$, as determined by Alizarin red staining of matrix calcium (Figure $3 \mathrm{~A}$ ) and total calcium quantification in cell lysates measured by the Arsenazo III method (Figure 3B); such calcification did not occur when potassium levels were maintained at $5.7 \mathrm{mM}$ and $6.0 \mathrm{mM}$.

We and others have demonstrated that VSMC calcification resembles osteogenic differentiation of bone cells $(38,39)$; therefore, we determined the effect of potassium on the osteogenic differentiation of VSMCs, as indicated by the changes in the expression of bone markers and smooth muscle markers (13, 38). Increased expression of Runx 2 , a key osteogenic transcription factor that we have determined to be an important regulator of VSMC calcification $(13,38)$, was found to be upregulated by low potassium at both protein and mRNA levels (Figure 3, C and D). Consistently, the expression of Runx2-regulated osteogenic markers, including osteocalcin (OC) and alkaline phosphatase (ALP), were induced (Figure 3D), while the SMC marker genes, $\alpha$-smooth muscle actin ( $\alpha$-SMA) and smooth muscle protein $22 \alpha$ (SM22 $\alpha$ ), were markedly reduced concurrently (Figure 3, C and E). These data indicated a direct effect of low potassium 
A

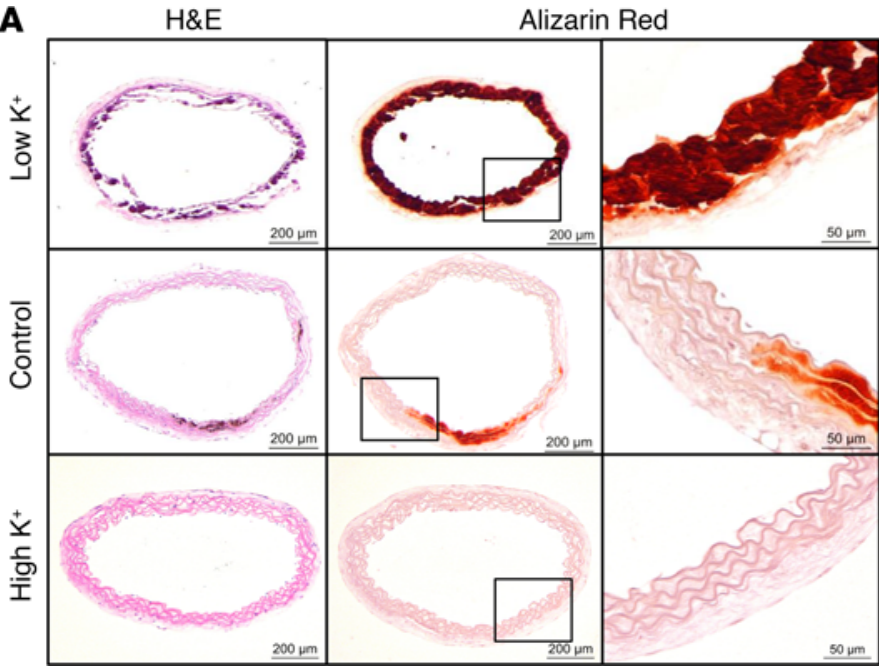

B

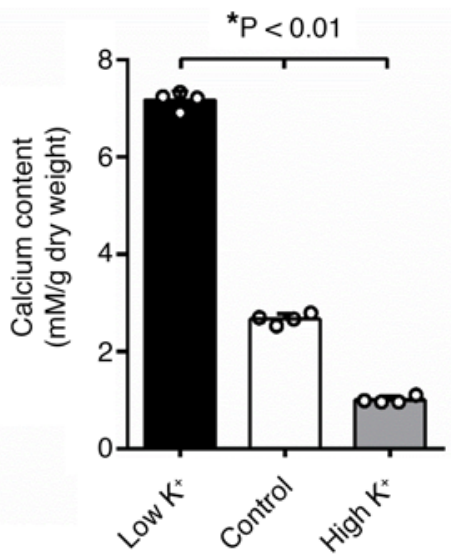

Figure 2. Potassium regulated vascular calcification ex vivo. Aortic rings prepared from wild-type mice were exposed to osteogenic media with control $(5.4 \mathrm{mM})$, low potassium $\left(3.7 \mathrm{mM}\right.$, Low $\left.\mathrm{K}^{+}\right)$, or high potassium $\left(6.0 \mathrm{mM}\right.$, High $\left.\mathrm{K}^{+}\right)$for 3 weeks. (A) Aortic calcification, determined in consecutive sections of the aortic rings by Alizarin red staining (middle panels). H\&E staining (left panels) was performed for histology. Higher-magnification images of the boxed areas in the middle panels are shown in the right panels. Representative images from 4 independent experiments performed in duplicate are shown. Scale bars: $200 \mu \mathrm{m}$ (left and middle panels) and $50 \mu \mathrm{m}$ (right panels). (B) Total calcium content of aortic rings in each group determined by Arsenazo III in separated sets of experiments. Results shown are normalized by total protein amount. Bar values are means $\pm S D\left(n=4,{ }^{*} P<0.01\right)$. Statistical analysis was performed by 1 -way ANOVA followed by a Student-Newman-Keuls test.

on promoting VSMC osteogenic differentiation and calcification, via increasing osteogenic markers and decreasing SMC markers.

Activation of intracellular calcium signaling mediated low-potassium-induced VSMC calcification. Potassium deficiency has been shown to increase intracellular calcium in VSMCs (40). As increased intracellular calcium flux has been linked to VSMC calcification (41), we examined whether low potassium may induce VSMC calcification via activation of calcium signaling. We found that potassium at a control condition of $5.4 \mathrm{mM}$ did not affect calcium flux (Figure 4A, dotted line). In contrast, elevation of intracellular calcium was evident in VSMCs within minutes after exposure to a low potassium concentration of $3.7 \mathrm{mM}$; the increase was sustained over the 30-minute duration (Figure 4A). Consistently, low potassium induced rapid and heightened activation of several known downstream mediators that include protein kinase C (PKC), and calcium-activated CREB (Figure 4B), but did not affect extracellular signal-regulated kinases (ERKs) signaling.

As potassium can be transported via 4 types of potassium channels (42), we determined the roles of these channels in mediating low-potassium-induced VSMC calcification. We found that inhibition of ATP-sensitive potassium channels $\left(\mathrm{K}_{\mathrm{ATP}}\right)$ by glibenclamide, voltage-dependent potassium channels $(\mathrm{Kv})$ by 4 -aminopyridine, and calcium-activated potassium channels $\left(\mathrm{K}_{\mathrm{Ca}}\right)$ by TRAM34 and charybdotoxin had no effects (Supplemental Figure 2), while inhibition of inward-rectifier potassium channels $\left(\mathrm{K}_{\mathrm{IR}}\right)$ by barium $(43,44)$ abolished low-potassium-induced VSMC calcification (Figure 4C). Furthermore, inhibition of potassium-activated calcium signaling by calcium channel inhibitors, verapamil and nifedipine, attenuated low-potassium-induced vascular calcification (Figure 4C). Altogether, these results showed that lowpotassium-induced elevation of intracellular calcium signaling mediated its effects on VSMC calcification.

Activation of calcium signaling-mediated CREB was required for low potassium-induced VSMC calcification. The effects of these inhibitors of potassium or calcium channels on calcium signaling-activated downstream signal CREB were further determined. Consistent with the inhibitory effects on low-potassium-induced VSMC calcification, the low-potassium-induced activation of CREB in VSMCs was abolished by the $\mathrm{K}_{\mathbb{I R}}$ inhibitor, barium, as well as the calcium channel inhibitors, verapamil and nifedipine (Figure 4D). Furthermore, knockdown of CREB using lentivirus-mediated short hairpin RNA (shCREB) blocked low-potassium-induced VSMC calcification and Runx2 upregulation (Figure 4, E and F), supporting the requirement of intracellular calcium-activated CREB signaling in mediating low-potassium-induced osteogenic differentiation and calcification of VSMCs. 
A

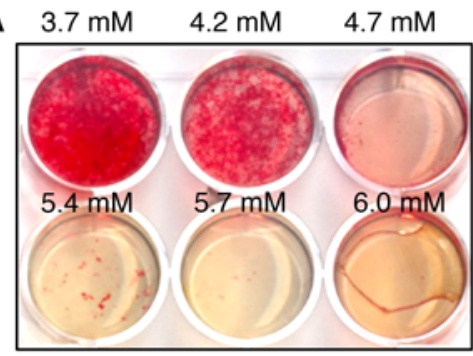

D

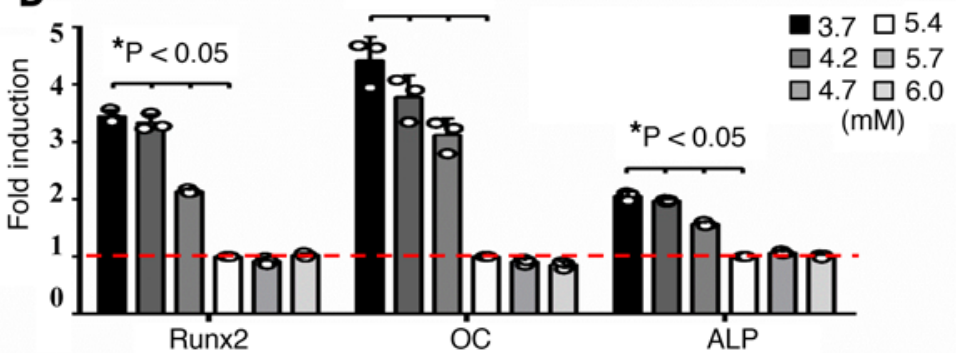

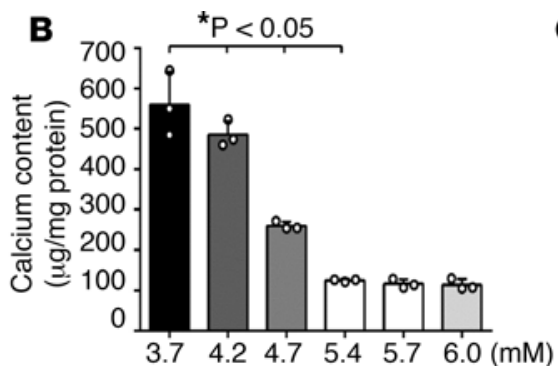

$\begin{array}{llllll}3.7 & 4.2 & 4.7 & 5.4 & 5.7 & 6.0(\mathrm{mM})\end{array}$

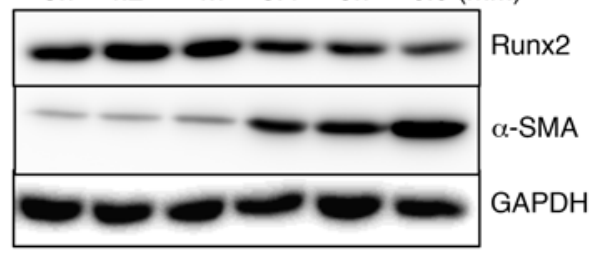

$\mathbf{E}$

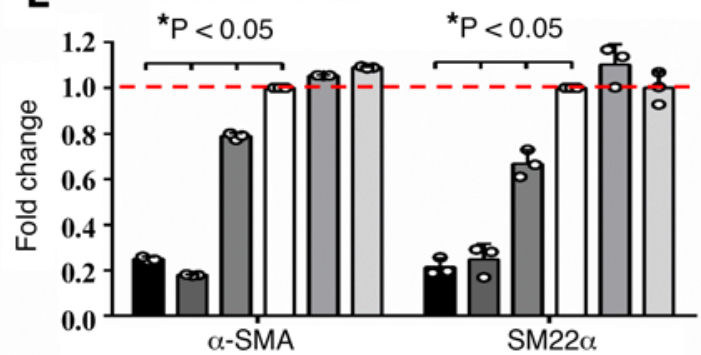

Figure 3. Lower physiological concentrations of potassium induced VSMC osteogenic differentiation and calcification. (A) Effects of potassium levels on calcification of vascular smooth muscle cells (VSMCs), determined by Alizarin red staining. VSMCs were cultured in osteogenic media with increased concentrations of potassium, 3.7 to $6.0 \mathrm{mM}$, for 3 weeks. Representative images of stained dishes from 4 independent experiments are shown. (B) Total calcium content in VSMCs, determined by Arsenazo III. VSMCs were cultured in osteogenic media with increased concentrations of potassium, 3.7 to 6.0 $\mathrm{mM}$, for 3 weeks. Results shown are normalized by total protein amount. Bar values are means $\pm \mathrm{SD}\left(n=3,{ }^{*} P<0.05\right.$ compared with potassium at 5.4 mM). (C) Effects of potassium levels on the expression of osteogenic and smooth muscle cell markers. VSMCs were exposed to 3.7 to $6.0 \mathrm{mM}$ of potassium for 3 weeks. Representative images of Western blot analysis of runt-related transcription factor 2 (Runx2) and $\alpha$-smooth muscle actin ( $\alpha$-SMA) proteins in VSMCs exposed to different concentrations of potassium from 3 independent experiments are shown. (D and $\mathbf{E}$ ) Real-time PCR analysis of (D) osteogenic markers, Runx2, osteocalcin (OC), and alkaline phosphatase (ALP) and (E) smooth muscle cell markers, $\alpha$-SMA and smooth muscle protein $22 \alpha$ (SM22 $\alpha$ ). VSMCs were exposed to 3.7 to $6.0 \mathrm{mM}$ potassium for 10 days. Results from 3 independent experiments performed in duplicate are shown. Bar values are means $\pm S D$ ( ${ }^{*} P$ $<0.05$ compared with potassium at $5.4 \mathrm{mM}$ ). Statistical analysis was performed by 1-way ANOVA followed by a Student-Newman-Keuls test.

As activation of CREB signaling was linked to increased autophagy in human melanoma cells and liver tissues $(45,46)$ and elevation of autophagy was demonstrated during osteoblast mineralization (47), we determined whether CREB activation may affect autophagy in VSMCs during low-potassium-induced calcification. We found that low potassium induced the microtubule-associated protein 1 light chain 3 in the conjugated form (LC3 II) (Figure 4F), an indicator of autophagy activation (48). CREB knockdown, however, markedly inhibited low-potassium-induced elevation of LC3 II (Figure 4F). Consistently, inhibition of $\mathrm{K}_{\mathrm{IR}}$ or calcium channels that were upstream of CREB by barium, verapamil, and nifedipine also attenuated low-potassium-induced expression of LC3 II (Figure 4G), implying that intracellular calciumactivated CREB mediates low-potassium-induced VSMC calcification via the regulation of autophagy.

Low potassium promoted VSMC calcification through autophagy. To determine the role of autophagy in lowpotassium-induced VSMC calcification, we first assessed the effects of potassium levels on the expression of LC3 II. Increased expression of LC3 II (Figure 5A) was demonstrated in VSMCs exposed to potassium at concentrations that induced VSMC calcification (Figure 3A). Quantitative analysis further confirmed an increased LC3 II/I ratio by low potassium (Figure 5B), indicating that low potassium activated autophagy in VSMCs. In addition, a time-dependent effect of low potassium, at $3.7 \mathrm{mM}$, in increasing the LC3 II/I ratio and autophagy activation was demonstrated (Supplemental Figure 3).

The essential role of autophagy in mediating low-potassium-induced VSMC calcification was initially determined utilizing 3-methyladenine (3-MA), a pharmacological inhibitor of autophagy. Pretreatment of VSMCs with 3-MA dramatically reduced low-potassium-induced elevation of the LC3 II/I ratio, and concurrently blocked VSMC calcification (Figure 5, C and D). Moreover, we generated stable VSMCs with shRNA knockdown of ATG7, a key regulator of autophagy formation. Similar to the observations with the autophagy inhibitor, the ATG7 knockdown blocked VSMC calcification and inhibited low-potassiuminduced elevation of the LC3 II/I ratio (Figure 5, E and F). These results support a definitive role of lowpotassium-induced autophagy in mediating its effects on promoting VSMC calcification. 

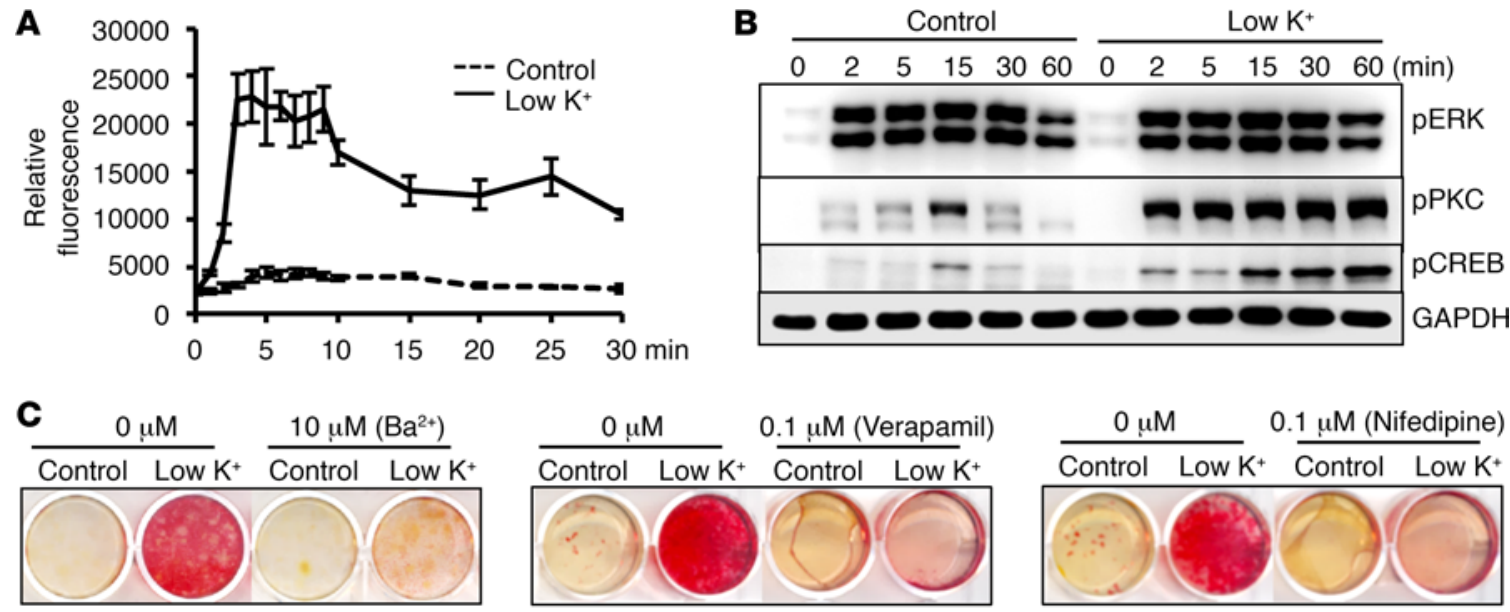

\section{D}

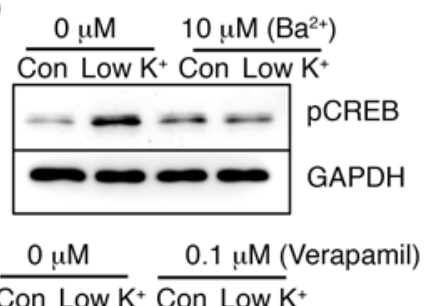

E

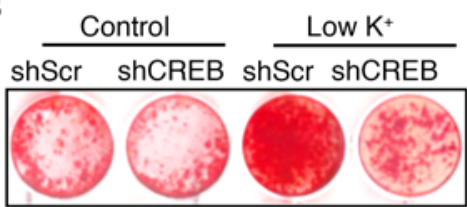

G $\frac{0 \mu \mathrm{M}}{\operatorname{Con~Low~K}} \frac{10 \mu \mathrm{M}\left(\mathrm{Ba}^{2+}\right.}{\operatorname{Con~Low~K}}$
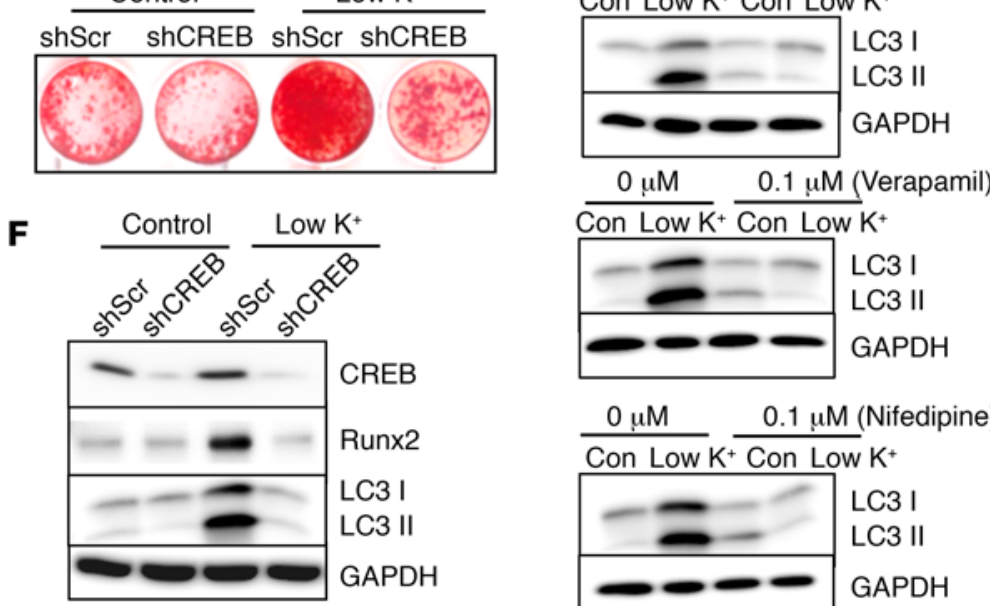

Con Low K + Con Low $\mathrm{K}^{+}$

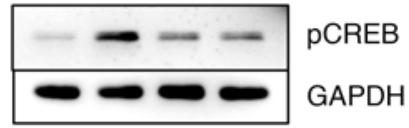

$0 \underline{\mu \mathrm{M}} \quad 0.1 \mu \mathrm{M}$ (Nifedipine)

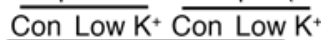

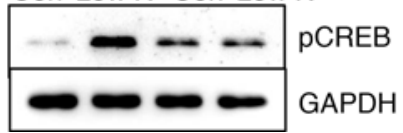

GAREB

Figure 4. Activation of calcium signaling-mediated CREB was required for low-potassium-induced VSMC calcification. (A) Effects of potassium levels on intracellular calcium, determined by Fluo4 NW, in vascular smooth muscle cells (VSMCs) exposed to control (5.4 mM) or low potassium (3.7 mM, Low $\left.\mathrm{K}^{+}\right)$. Results from 3 independent experiments are shown. (B) Effects of low potassium on activation of extracellular signal-regulated kinase (ERK), protein kinase C (PKC), and calcium-activated CAMP response element-binding protein (CREB), determined by Western blot analysis. Representative blots from 3 independent experiments are shown. (C) Effects of pharmacological inhibitors on VSMC calcification. VSMCs were exposed to control or low-potassium media with the indicated inhibitors for 3 weeks. Calcification was determined by Alizarin red staining. (D) Effects of pharmacological inhibitors on activation of CREB. Western blot analysis of phosphorylation of CREB in C. Representative images from 3 independent experiments are shown. (E and $\mathbf{F}$ ) Effects of CREB knockdown on low-potassium-induced VSMC calcification. VSMCs with CREB knockdown by shRNA (shCREB) or control shRNA (shScr) were exposed to control or low-potassium media for 3 weeks. Calcification was determined by Alizarin red staining. Western blot analysis was performed to determine the expression of CREB, runt-related transcription factor 2 (Runx2), and the autophagic marker, microtubule-associated protein 1 light chain 3 (LC3), in the cytoplasmic form (LC3 I), and conjugated form (LC3 II). Representative blots from 3 independent experiments are shown. (C) Effects of pharmacological inhibitors on autophagy markers. Western blot analysis of LC3 I and II levels in VSMCs exposed to control or low-potassium media for 3 weeks, in the presence or absence of indicated inhibitors. Representative results from 3 independent experiments are shown.

Potassium regulated the activation of $C R E B$ and autophagy in vascular calcification ex vivo and in vivo. The roles of activation of CREB and autophagy signals in low-potassium-induced vascular calcification were further determined in the ex vivo ring culture model and in vivo mouse models. As shown in Figure 6A, low-potassium-induced aortic calcification ex vivo was associated with increased activation of intracellular calciumactivated signals, including PKC, CREB, and elevation of LC3 II as well as upregulation of the osteogenic transcription factor Runx2 (Figure 6A). In contrast, high potassium markedly inhibited the activation of PKC and CREB and inhibited LC3 II and Runx2 (Figure 6A). Moreover, increased activation of PKC, CREB, and elevation of LC3 II and Runx2 were demonstrated in arteries from low dietary potassium-fed mice (Figure 6B). Consistently, higher dietary potassium inhibited PKC and CREB activation, and decreased LC3 II and Runx2 (Figure 6B). Altogether, these results further supported an important role for potassium in regulating vascular calcification via effects on calcium signaling, CREB, and autophagy in VSMCs (Figure 6C). 

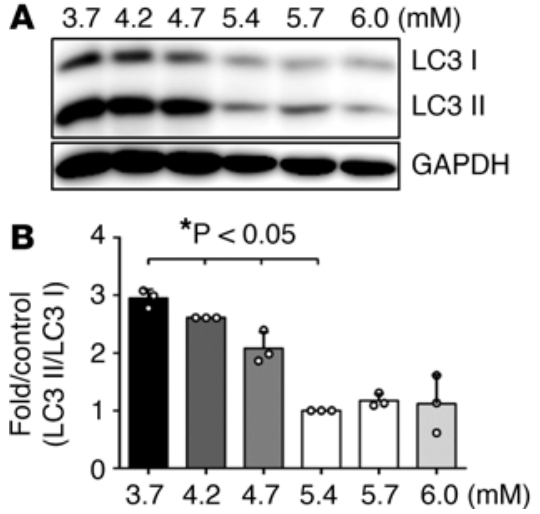
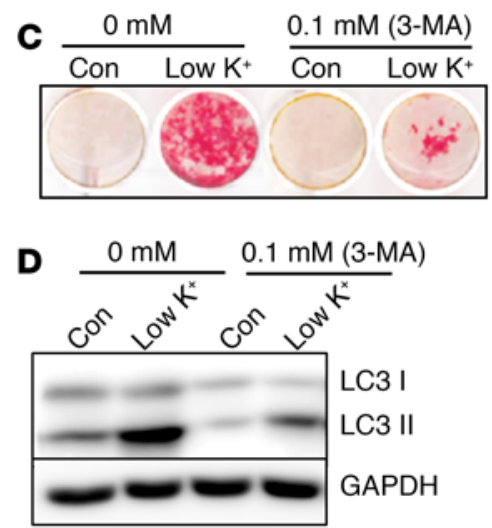
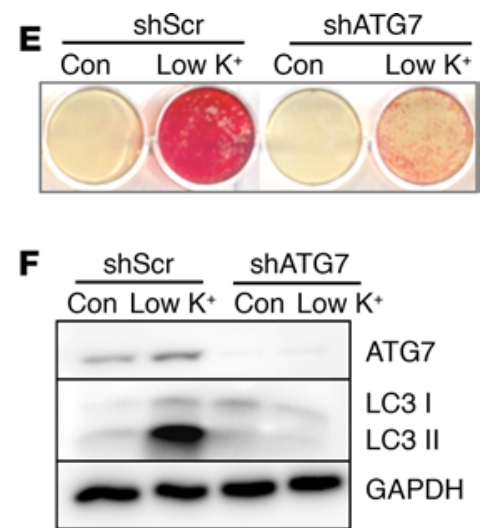

Figure 5. Low potassium promoted VSMC calcification through autophagy. (A) Effects of potassium concentrations on autophagy in vascular smooth muscle cells (VSMCs). VSMCs were cultured in osteogenic media with increased concentrations of potassium for 3 weeks. Western blot analysis was performed to determine the expression of the autophagic marker, microtubule-associated protein 1 light chain 3 (LC3), in the cytoplasmic form (LC3 I) and conjugated form (LC3 II). Representative blots from 3 independent experiments are shown. (B) Quantitative analysis of the LC3 II/I ratio in A, by densitometric analysis using Image) software. Bar values are means $\pm \mathrm{SD}\left(n=3,{ }^{*} P<0.05\right.$ compared with potassium at 5.4 mM). Statistical analysis was performed by 1-way ANOVA followed by a Student-Newman-Keuls test. (C) Effects of inhibition of autophagy on low-potassium-induced VSMC calcification. VSMCs were exposed to osteogenic media with control or low potassium for 3 weeks, with or without the autophagy inhibitor, 3-methyladenine (3-MA). Calcification was determined by Alizarin red staining. (D) Effects of 3-MA on autophagy markers. Western blot analysis was used to determine LC3 I and II levels in C. Representative blots from 3 independent experiments are shown. (E) Effects of knockdown of autophagy-related 7 protein (ATC7) on low-potassiuminduced VSMC calcification. VSMCs with ATG7 stably knocked down by shRNA (shATG7) or with control shRNA (shScr) were exposed to osteogenic media with control or low potassium for 3 weeks. Calcification was determined by Alizarin red staining. (F) Effects of ATC7 knockdown on autophagy markers. Western blot analysis was used to determine ATG7 and LC3 I and II levels in E. Representative results from 3 independent experiments are shown.

\section{Discussion}

Reduced dietary potassium intake has been linked to the pathogenesis of a variety of human diseases, including atherosclerosis, diabetes, and chronic kidney disease; all of these disease share common vascular complications, such as vascular calcification. Using in vitro cell culture, an ex vivo tissue model, and in vivo atherogenic animals, we have demonstrated that low potassium intake induces VSMC osteogenic differentiation and calcification and promotes atherosclerotic vascular calcification. Concurrently with increased vascular calcification, we have found that PWV, an indicator of arterial stiffness, was increased in animals fed a low-potassium diet (Figure 1D), supporting a direct link between low dietary potassium-promoted atherosclerotic calcification and arterial stiffness. These observations are consistent with previous studies demonstrating that vascular calcification may increase arterial stiffness through the calcification of elastic fibers $(49,50)$. In sharp contrast, increased dietary potassium inhibited atherosclerotic calcification and improved vascular stiffness in the $A p o E^{-/-}$mice. Importantly, the increase in vascular calcification and stiffness caused by the reduced-potassium diet occurred with only a small reduction in mean serum potassium concentration, compared with the standard potassium-content $\operatorname{diet}(3.70 \pm 0.21$ versus $4.27 \pm 0.23 \mathrm{mEq} / 1$; $P<0.05)$. Similarly, increased potassium diets resulted in a small increase in serum potassium concentration $(4.73 \pm 0.15 \mathrm{mEq} / 1 ; P<0.05)$ and was sufficient to reduce vascular calcification in atherosclerosis. Of note, lower magnesium levels have been reported to be associated with the presence of vascular calcification in clinics (51), as well as in calcification of cultured VSMCs (52). We did not find significant differences in the serum magnesium levels in the low-potassium-fed animals compared with normal- or highpotassium-fed animals (Supplemental Table 1). Therefore, results from these studies support a direct effect of potassium on the calcification of VSMCs, leading to vascular calcification and stiffness. These findings are consistent with previous observations that reduced dietary intake of potassium is associated with increased risk of vascular diseases, such as stroke and hypertension, which were improved by appropriate dietary potassium intake $(22-24,53)$. These studies uncovered a potentially novel causative link between low potassium and VSMC osteogenic differentiation and calcification in atherosclerosis.

Our studies have added mechanistic insights into our current understanding of the regulation of vascular health and disease by dietary potassium. During the development of atherosclerosis, potassium may regulate multiple cellular processes, as it can affect VSMC proliferation (37), or interplay with sodium homeostasis and a number of neurohormonal pathways that regulate blood pressure (54). Our findings highlight the importance of dietary potassium-regulated VSMC calcification in atherosclerosis. 

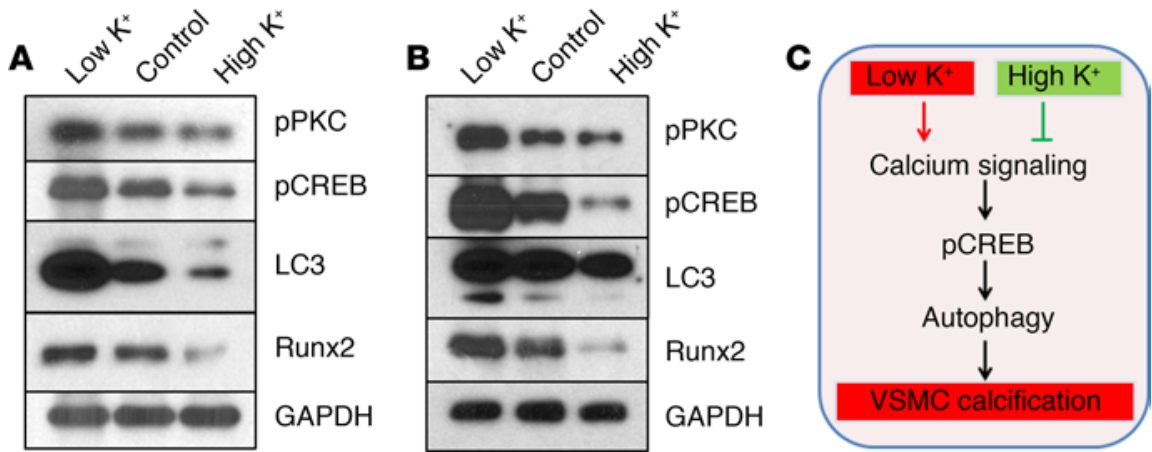

Figure 6. Potassium regulated the activation of CREB and autophagy in vascular calcification ex vivo and in vivo. (A) Effects of low potassium on the activation of calcium-activated cAMP response element-binding protein (CREB) and autophagy signals ex vivo. Aortic rings from wild-type mice were exposed to osteogenic media with control (5.4 $\mathrm{mM}$ ), low potassium (3.7 mM, Low $\left.\mathrm{K}^{+}\right)$, or high potassium $\left(6.0 \mathrm{mM}\right.$, High $\left.\mathrm{K}^{+}\right)$for 3 weeks. Western blot analysis was performed to determine the phosphorylation of protein kinase C (PKC), CREB, and the expression of microtubuleassociated protein 1 light chain 3 (LC3) and runt-related transcription factor 2 (Runx2). Representative blots from 3 independent experiments are shown. (B) Effects of dietary potassium on the activation of CREB and autophagy signals in vivo. Western blot analysis was performed to determine the phosphorylation of PKC, CREB and the expression of LC3 and Runx 2 in descending aortas of control, low, or high potassium diet-fed mice. Representative Western blot analyses of protein lysates extracted from the descending aortas from at least 3 mice in each group are shown. (C) Schematic illustration of vascular calcification regulated by potassium. Low potassium activates calcium signaling that promotes activation of CREB, which results in chronically excessive autophagy in vascular smooth muscle cells (VSMCs), leading to the development of calcification. High potassium reverses vascular calcification.

The in vitro studies in particular demonstrated a direct effect of extracellular potassium levels on VSMC calcification. Although previous studies have shown that potassium reduced VSMC proliferation in vitro (25), the effect of low potassium on promoting VSMC calcification was independent of its effects on VSMC viability (Supplemental Figure 1), suggesting a previously unknown mechanism. Among the 4 known potassium channels on VSMCs, we identified that $\mathrm{K}_{\mathrm{IR}}$ specifically mediated the effects of lowpotassium-induced VSMC calcification. Upregulation of $\mathrm{K}_{\mathrm{IR}}$ has been reported during osteogenic differentiation of mesenchymal stromal cells; however, the role of $\mathrm{K}_{\mathrm{IR}}$ in the osteogenic process has not been defined (55). We found that low potassium initiated VSMC calcification through activation of intracellular calcium flux. As $\mathrm{K}_{\mathrm{IR}}$ was linked to potassium-induced changes in membrane potential of rat arteries (43), it is possible that $\mathrm{K}_{\mathrm{IR}}$-mediated membrane depolarization leads to the elevation of intracellular calcium via voltage-gated calcium channels (VGCCs) in VSMCs. In a meta-analysis combining the results of 2 genome-wide association studies, upregulation of CACNA1C, encoding a subunit of the VGCC, was associated with patients with calcific aortic valve disease (56), suggesting a role of VGCCs in vascular calcification. Furthermore, the VGCC blocker nifedipine was found to inhibit coronary arterial calcification in patients with hypertension (57). Consistently, we found that inhibitors of the $\mathrm{K}_{\mathrm{IR}}$ or VGCCs blocked low-potassium-induced VSMC calcification, which was associated with inhibition of low-potassium-induced calcium signals.

We observed a prolonged increment of intracellular calcium in low-potassium-treated VSMCs (Figure $4 \mathrm{~A})$, which was associated with increased VSMC calcification. Intracellular calcium influx resulting from depolarization-induced VGCC opening has been associated with SMC differentiation (58). However, a long-term increase in intracellular calcium is linked to osteogenic differentiation of SMCs, and inhibition of intracellular calcium intake by $\mathrm{K}_{\mathrm{Ca}}$ blocker attenuates calcium phosphate-induced VSMC calcification (59). In addition, inhibition of L-type calcium channels by verapamil has been shown to reduce VSMC calcification (60). Accordingly, differences in the duration of intracellular calcium flux may lead to diverse downstream molecular signals that differentially regulate VSMC physiology and pathology. Unlike physiologic intracellular calcium influx that is associated with excitation-contraction of SMCs, sustained increase in intracellular calcium may lead to VSMC pathology, such as calcification. Our results with the $\mathrm{K}_{\mathrm{IR}}$ or calcium channel inhibitors have further supported the role of calcium signaling in mediating lowpotassium-induced VSMC calcification.

Low-potassium-induced elevation of intracellular calcium led to the activation of CREB signaling, which was required for low-potassium-induced Runx2 upregulation and osteogenic differentiation and 
calcification of VSMCs. CREB is a target of cAMP signaling and is a widely expressed nuclear transcription factor that has been found to mediate the cellular response to metabolic and mitogenic signals (61). Activation of CREB signaling has been demonstrated in bone morphogenetic protein 9-induced osteogenic differentiation of mesenchymal stem cells (62). In addition, upregulation of CREB is associated with TNF- $\alpha$-enhanced calcification of bovine aortic SMCs in vitro (63). Results from our studies highlight an essential role of CREB in mediating low-potassium-induced VSMC calcification, as knockdown of CREB attenuated low-potassium-induced VSMC calcification.

Furthermore, we have established a link between CREB signaling and autophagy in VSMC calcification. Autophagy is an evolutionarily conserved, complex cellular pathway that delivers cytoplasmic contents for degradation in lysosomes $(64,65)$. Autophagosome-like structures are documented in VSMCs in atherosclerotic lesions in human and animal models (66). The present study has provided what we believe to be the first evidence that low potassium enhanced autophagy, which promoted VSMC osteogenic differentiation in vitro and vascular calcification in atherosclerosis in vivo. Autophagy has been shown to induce elastin degradation (67), which could lead to the initiation and progression of vascular calcification. Consistent with these observations, inhibition of autophagy by ATG7 knockdown or ATG14 knockout reduced osteogenic differentiation of human mesenchymal stem cells $(68,69)$, supporting the critical role of autophagy during osteogenic differentiation. The role of autophagy in regulating VSMC function appears to be complicated. For instance, inducing autophagy has been shown to protect statin-induced cell death of VSMCs in culture (70). On the other hand, excessive autophagy in VSMCs was associated with autophagic cell death of VSMCs and plaque destabilization in atherosclerosis $(71,72)$. Our studies found that low-potassium-induced autophagy in VSMCs did not affect VSMC viability, but promoted VSMC calcification. Accordingly, VSMC autophagy may contribute to atherosclerosis depending on disease status, location, and the surrounding microenvironment. In phosphate-induced VSMC calcification in vitro, autophagy has been shown to play an adaptive role (73-75). Inhibition of autophagy does not block high-phosphate-induced VSMC calcification, but increases calcium deposition and enhances vascular calcification. Our studies, however, have demonstrated that low-potassium-induced elevation of autophagy promotes vascular calcification in vitro and in vivo. Accordingly, different osteogenic stimuli and duration of autophagy may contribute to the varying responses of autophagy in the development of VSMC calcification in these 2 different systems. Of note, a low-potassium-induced LC3 II/I ratio in VSMCs was observed after 5 days and sustained even after 21 days of treatment (Supplemental Figure 3), suggesting that persistent activation of autophagy may promote VSMC calcification. It is conceivable that acute and chronic autophagy formation may regulate distinct underlying molecular mechanisms that promote VSMC calcification, further supporting the notion that autophagy plays a complex and often distinct role under various pathological conditions. Further investigations with animal models of tissue-specific knockout of autophagy components are warranted to define the role of autophagy in the pathogenesis of vascular calcification and stiffness in vivo. Nonetheless, our data suggest that the autophagic process may be a viable target to develop and design drugs that attenuate vascular calcification and stiffness in atherosclerosis.

In summary, we have determined a causative link between reduced dietary potassium and vascular calcification in atherosclerosis and uncovered the underlying pathogenic mechanisms that integrate enhanced intracellular calcium influx, activated CREB signaling, and elevation of autophagy. These findings provide molecular insights into the previously unappreciated regulation of vascular calcification and stiffness by low potassium intake and emphasize the need to consider dietary intake of potassium in the prevention of vascular complications of atherosclerosis.

\section{Methods}

Experimental animals. Effects of dietary potassium on pathogenesis of atherosclerotic vascular calcification were determined using the ApoE-deficient $\left(A p o E^{-/-}\right)$mouse model $(12,13)$. Eight-week-old male $A p o E^{-/-}$mice (The Jackson Laboratory) were fed a high-fat, high-cholesterol diet (HFD, Harlan Teklad diet TD88137) containing normal potassium $(0.7 \% \mathrm{wt} / \mathrm{wt} \mathrm{KCl})$, low potassium $(0.3 \% \mathrm{wt} / \mathrm{wt} \mathrm{KCl})$, or high potassium $(2.1 \% \mathrm{wt} / \mathrm{wt} \mathrm{KCl})$ for 30 weeks as previously described $(29,30)$. Both food and fluid intake were given ad libitum. The Institutional Animal Care and Use Committee of the University of Alabama at Birmingham approved all experimental protocols.

Echocardiography and measurement of $P W V$. At the experimental endpoint, echocardiography was performed with the high-resolution imaging system VEVO 2100 (Visual Sonics) to determine PWV as previously 
described (76). PWV was calculated as the distance divided by the time interval between the pulse wave at the aortic arch and the abdominal aorta $(\mathrm{m} / \mathrm{s})$.

Analysis of serum biochemical parameters. Mouse blood was collected at euthanization, and serum was separated from nonheparinized blood and assayed for various minerals, enzymes, and compounds (Supplemental Table 1). All the biochemical parameters were determined by Molecular Diagnostic Services, Inc. using a Beckman Coulter AU680 analyzer.

Tissue harvesting, processing, and analysis. Heart and aortic tissues were collected, processed, and analyzed as previously reported $(12,13,32,76)$. Descending aorta was used for total calcium measurement by the Arsenazo III method (Stanbio Laboratory), or protein expression and phosphorylation by Western blot analysis. A series of consecutive 7- $\mu \mathrm{m}$ aortic root sections were used for hematoxylin and eosin (H\&E) staining for histology and Alizarin red staining (Sigma-Aldrich) for calcification. Stained sections were examined using a Leica M165 FC microscope, and the percentage of positively stained area in each section was quantified using ImageJ software (NIH). All quantitative analyses were performed blindly by at least 2 investigators.

Ex vivo aortic calcification. Aortic rings were obtained from descending aortas of wild-type C57BL/6 mice (The Jackson Laboratory) as we previously described (32). Calcification of aortic rings was performed as previously reported (32), using 3-mm mouse aortic rings cultured in osteogenic media containing $1 \%$ FBS with low $(3.7 \mathrm{mM})$, normal $(5.4 \mathrm{mM})$, or high $(6.0 \mathrm{mM})$ potassium for up to 3 weeks. Choline chloride, which has been used to compensate for the deficit in the extracellular ionic concentration (77-79) and does not show any effects on vascular calcification (80), was added to culture media to avoid the changes in osmolarity. Total calcium content in the aortic rings was quantified by the Arsenazo III method. The amount of calcium was normalized to the dry weight of the tissues and expressed as millimolar/gram dry weight. In separate experiments, aortic rings were fixed in $4 \%$ paraformaldehyde, paraffin embedded, and consecutive $7-\mu \mathrm{m}$ sections were used for H\&E and Alizarin red staining as we previously reported $(12,13,32)$.

VSMC culture and in vitro VSMC calcification. Primary VSMCs were isolated from descending aortas from wild-type C57BL/6 mice and cultured in growth media as described previously $(13,32,38,76)$. All experiments were performed with VSMCs at passages 3 to 5. Potassium-free DMEM (10-013-CM) was purchased from Mediatech Inc. The potassium concentration in the culture media, from $3.7 \mathrm{mM}$ to 6.0 $\mathrm{mM}$, was adjusted with potassium chloride $(\mathrm{KCl})$ (Sigma-Aldrich). Choline chloride was added to culture media to avoid the changes in osmolarity of low-potassium media.

To determine the effects of potassium level on VSMC calcification, VSMCs were cultured in osteogenic media containing $0.25 \mathrm{mmol} / 1 \mathrm{~L}$-ascorbic acid, $10 \mathrm{mmol} / 1 \beta$-glycerophosphate, and $10^{-8} \mathrm{M}$ dexamethasone with different concentrations of potassium for 3 weeks. Calcification was determined by Alizarin red staining and quantified by measuring total calcium using Arsenazo III as previously described $(13,32,38,76)$.

The effects of pharmacological inhibitors on VSMC calcification were determined by addition of nontoxic amounts of the selective inhibitors in the osteogenic media: L-type VGCC inhibitors, verapamil and nifedipine (Calbiochem); $\mathrm{K}_{\mathrm{IR}}$ inhibitor, barium chloride $\left(\mathrm{BaCl}\right.$ ) (Sigma-Aldrich); $\mathrm{K}_{\mathrm{ATP}}$ inhibitor, glibenclamide (Tocris); Kv inhibitor, 4-aminopyridine (Tocris); $\mathrm{K}_{\mathrm{Ca}}$ inhibitors, TRAM34 and charybdotoxin (Tocris); and autophagy inhibitor, 3-MA (Tocris).

VSMC viability. VSMCs were grown in a 96-well plate at $3 \times 10^{3}$ cells per well for 72 hours in osteogenic medium containing extracellular potassium, ranging from $1 \mathrm{mM}$ to $108 \mathrm{mM}$. The effects of potassium on VSMC viability was determined by MTS assay, using the MTS Cell Proliferation Assay Kit (Promega) as described previously (81).

Real-time polymerase chain reaction (PCR). The expression of osteogenic and smooth muscle markers in VSMCs was determined by real-time PCR as described previously $(12,13,32,38,76)$. Total RNA was isolated using TRIzol (Invitrogen) and reverse transcribed into cDNA. SYBR Green-based real-time PCR was performed using specific primers for Runx2, murine ALP, OC, $\alpha$-SMA, and SM22 $\alpha$, using SsoFast EvaGreen Supermix (Bio-Rad) on a C1000 Thermal Cycler (Bio-Rad).

Western blot analysis. Protein extraction, protein concentration measurement, and polyacrylamide gel electrophoresis were performed as previously reported (38). Western blot analysis was performed using specific antibodies for Runx2 (MBL, D130-3), $\alpha$-SMA (Sigma-Aldrich, A2547), GAPDH (Fitzgerald, 10R-G109a), p-ERK (Cell Signaling, 4370), p-PKC (Cell Signaling, 9375), p-CREB (Cell Signaling, 9198), LC3 (MBL, M186-3), and total CREB (Cell Signaling, 9104), and ATG7 (Cell Signaling, 8558); and detected with a chemiluminescence detection kit (Millipore). 
Intracellular calcium measurement. Intracellular calcium was measured using a Fluo4 NW calcium assay kit (Molecular Probes) according to the manufacturer's instructions. Briefly, VSMCs seeded on 96-well black clear-bottom plates (Corning Costar) were preloaded with Fluo4 NW fluorescent dye $(1 \mu \mathrm{M})$ for 30 minutes, and then exposed to growth media containing low $(3.7 \mathrm{mM})$ or normal potassium $(5.4 \mathrm{mM})$. Fluorescence signals were recorded for 30 minutes at 1 -minute intervals using the fluorescence microplate reader with excitation at $485 \mathrm{~nm}$ and emission at $525 \mathrm{~nm}$ (Synergy 2, BioTek). Intracellular calcium was calculated by subtracting the basal fluorescence intensity from the total fluorescence intensity.

shRNA knockdown in VSMCs. Lentiviral vector carrying shRNA specific for murine CREB, ATG7, or control scrambled shRNA (shScr) was purchased from Open Biosystems and packaged into lentiviral particles as previously described (12). Lentiviral transduction was performed by incubating VSMCs with lentiviruses in growth media supplemented with $8 \mu \mathrm{g} / \mathrm{ml}$ polybrene, and stable transfectants were selected with puromycin $(2 \mu \mathrm{g} / \mathrm{ml})$ for 2 weeks.

Statistics. All the data are expressed as means \pm SD. For multiple group comparison, 1-way analysis of variance (ANOVA) followed by a Student-Newman-Keuls test was performed. A $P$ value less than 0.05 was considered statistically significant.

Study approval. All animal experiments were performed according to protocols approved by Institutional Animal Care and Use Committee at the University of Alabama at Birmingham.

\section{Author contributions}

HW and YC designed the study. YS, CHB, YY, and WEB performed the experiments and analyzed the data. YS, CHB, HW, and YC wrote the manuscript. LJD, PWS, and AA participated in study designing and modification, and edited the manuscript.

\section{Acknowledgments}

We thank Jay McDonald, University of Alabama at Birmingham for critical review and helpful discussion. This work was supported in part by NIH grants HL092215, HL136165, and DK100847 (to YC) and DK079337 (to AA), as well as Veterans Affairs Research Department grants BX000369 and BX003617 (to YC) and BX001591 (to PWS, YC, and AA).

Address correspondence to: Yabing Chen, 1825 University Blvd., 614 Shelby Biomedical Research Building, Birmingham, Alabama 35294, USA. Phone: 205.996.6293; Email: yabingchen@uabmc.edu.

1. O'Leary DH, Polak JF, Kronmal RA, Manolio TA, Burke GL, Wolfson SK. Carotid-artery intima and media thickness as a risk factor for myocardial infarction and stroke in older adults. Cardiovascular Health Study Collaborative Research Group. NEngl $J$ Med. 1999;340(1):14-22.

2. Lakatta EG, Levy D. Arterial and cardiac aging: major shareholders in cardiovascular disease enterprises: Part I: aging arteries: a "set up" for vascular disease. Circulation. 2003;107(1):139-146.

3. Laurent S, Boutouyrie P. Recent advances in arterial stiffness and wave reflection in human hypertension. Hypertension. 2007;49(6):1202-1206.

4. Payne RA, Wilkinson IB, Webb DJ. Arterial stiffness and hypertension: emerging concepts. Hypertension. 2010;55(1):9-14.

5. Jung $\mathrm{CH}$, et al. The relationship between coronary artery calcification score, plasma osteoprotegerin level and arterial stiffness in asymptomatic type 2 DM. Acta Diabetol. 2010;47(suppl 1):145-152.

6. McEniery CM, et al. Aortic calcification is associated with aortic stiffness and isolated systolic hypertension in healthy individuals. Hypertension. 2009;53(3):524-531.

7. Toussaint ND, Lau KK, Strauss BJ, Polkinghorne KR, Kerr PG. Relationship between vascular calcification, arterial stiffness and bone mineral density in a cross-sectional study of prevalent Australian haemodialysis patients. Nephrology (Carlton). 2009;14(1):105-112.

8. Ford ML, Tomlinson LA, Smith ER, Rajkumar C, Holt SG. Fetuin-A is an independent determinant of change of aortic stiffness over 1 year in non-diabetic patients with CKD stages 3 and 4. Nephrol Dial Transplant. 2010;25(6):1853-1858.

9. Mizobuchi M, Towler D, Slatopolsky E. Vascular calcification: the killer of patients with chronic kidney disease. $J$ Am Soc Nephrol. 2009;20(7):1453-1464.

10. Giachelli CM. Vascular calcification mechanisms. J Am Soc Nephrol. 2004;15(12):2959-2964.

11. Shao JS, Cai J, Towler DA. Molecular mechanisms of vascular calcification: lessons learned from the aorta. Arterioscler Thromb Vasc Biol. 2006;26(7):1423-1430.

12. Byon $\mathrm{CH}$, et al. Runx2-upregulated receptor activator of nuclear factor $\kappa \mathrm{B}$ ligand in calcifying smooth muscle cells promotes migration and osteoclastic differentiation of macrophages. Arterioscler Thromb Vasc Biol. 2011;31(6):1387-1396.

13. Sun Y, et al. Smooth muscle cell-specific runx2 deficiency inhibits vascular calcification. Circ Res. 2012;111(5):543-552. 
14. Steitz SA, et al. Smooth muscle cell phenotypic transition associated with calcification: upregulation of Cbfa1 and downregulation of smooth muscle lineage markers. Circ Res. 2001;89(12):1147-1154.

15. Watson KE, Parhami F, Shin V, Demer LL. Fibronectin and collagen I matrixes promote calcification of vascular cells in vitro, whereas collagen IV matrix is inhibitory. Arterioscler Thromb Vasc Biol. 1998;18(12):1964-1971.

16. Hou G, Vogel W, Bendeck MP. The discoidin domain receptor tyrosine kinase DDR1 in arterial wound repair. J Clin Invest. 2001;107(6):727-735.

17. Toussaint ND, Lau KK, Strauss BJ, Polkinghorne KR, Kerr PG. Relationship between vascular calcification, arterial stiffness and bone mineral density in a cross-sectional study of prevalent Australian haemodialysis patients. Nephrology (Carlton). 2009;14(1):105-112.

18. Korgaonkar S, et al. Serum potassium and outcomes in CKD: insights from the RRI-CKD cohort study. Clin J Am Soc Nephrol. 2010;5(5):762-769.

19. Sun K, et al. Serum potassium level is associated with metabolic syndrome: a population-based study. Clin Nutr. 2014;33(3):521527.

20. Ribeiro SC, Figueiredo AE, Barretti P, Pecoits-Filho R, de Moraes TP, all centers that contributed to BRAZPD II study. Low serum potassium levels increase the infectious-caused mortality in peritoneal dialysis patients: a propensity-matched score study PLoS One. 2015;10(6): 0127453.

21. Lai YH, Leu HB, Yeh WT, Chang HY, Pan WH. Low-normal serum potassium is associated with an increased risk of cardiovascular and all-cause death in community-based elderly. J Formos Med Assoc. 2015;114(6):517-525.

22. Whelton PK, et al. Effects of oral potassium on blood pressure. Meta-analysis of randomized controlled clinical trials. JAMA 1997;277(20):1624-1632.

23. Geleijnse JM, Kok FJ, Grobbee DE. Blood pressure response to changes in sodium and potassium intake: a metaregression analysis of randomised trials. J Hum Hypertens. 2003;17(7):471-480.

24. Report of the joint WHO/FAO expert consultation. Diet, nutrition the prevention of chronic diseases. World Health Organ Tech Rep Ser. 2003;916:i-viii, 1-149, backcover.

25. Landmark K. [Hypokalemia can accelerate the development of cerebrovascular and cardiovascular disease]. Tidsskr Nor Laegeforen. 2002;122(5):499-501.

26. Ma G, Mason DP, Young DB. Inhibition of vascular smooth muscle cell migration by elevation of extracellular potassium concentration. Hypertension. 2000;35(4):948-951.

27. Ma G, Young DB, Clower BR, Anderson PG, Lin H, Abide AM. High potassium intake inhibits neointima formation in the rat carotid artery balloon injury model. Am J Hypertens. 2000;13(9):1014-1020.

28. Young DB, Lin H, McCabe RD. Potassium's cardiovascular protective mechanisms. Am J Physiol. 1995;268(4 pt 2):R825-R837.

29. Ying WZ, Aaron K, Wang PX, Sanders PW. Potassium inhibits dietary salt-induced transforming growth factor- $\beta$ production. Hypertension. 2009;54(5):1159-1163.

30. Ying WZ, Aaron KJ, Sanders PW. Sodium and potassium regulate endothelial phospholipase C- $\gamma$ and Bmx. Am J Physiol Renal Physiol. 2014;307(1):F58-F63.

31. Willum-Hansen T, et al. Prognostic value of aortic pulse wave velocity as index of arterial stiffness in the general population. Circulation. 2006;113(5):664-670.

32. Deng L, Huang L, Sun Y, Heath JM, Wu H, Chen Y. Inhibition of FOXO1/3 promotes vascular calcification. Arterioscler Thromb Vasc Biol. 2015;35(1):175-183.

33. Shroff RC, et al. Chronic mineral dysregulation promotes vascular smooth muscle cell adaptation and extracellular matrix calcification. J Am Soc Nephrol. 2010;21(1):103-112.

34. Traslavina RP, et al. Euthanasia by $\mathrm{CO}_{2}$ inhalation affects potassium levels in mice. J Am Assoc Lab Anim Sci. 2010;49(3):316322.

35. Boehm O, et al. Clinical chemistry reference database for Wistar rats and C57/BL6 mice. Biol Chem. 2007;388(5):547-554.

36. Zhou X, Hansson GK. Effect of sex and age on serum biochemical reference ranges in C57BL/6J mice. Comp Med. 2004;54(2):176-178.

37. McCabe RD, Young DB. Potassium inhibits cultured vascular smooth muscle cell proliferation. Am J Hypertens. 1994;7(4 pt 1):346-350.

38. Byon $\mathrm{CH}$, et al. Oxidative stress induces vascular calcification through modulation of the osteogenic transcription factor Runx 2 by AKT signaling. J Biol Chem. 2008;283(22):15319-15327.

39. Mody N, Parhami F, Sarafian TA, Demer LL. Oxidative stress modulates osteoblastic differentiation of vascular and bone cells Free Radic Biol Med. 2001;31(4):509-519.

40. Adrogué HJ, Madias NE. Sodium and potassium in the pathogenesis of hypertension. N Engl J Med. 2007;356(19):1966-1978.

41. Rodenbeck SD, et al. Intracellular calcium increases in vascular smooth muscle cells with progression of chronic kidney disease in a rat model. Nephrol Dial Transplant. 2017;32(3):450-458.

42. Sobey CG. Potassium channel function in vascular disease. Arterioscler Thromb Vasc Biol. 2001;21(1):28-38.

43. Knot HJ, Zimmermann PA, Nelson MT. Extracellular K(+)-induced hyperpolarizations and dilatations of rat coronary and cerebral arteries involve inward rectifier K(+) channels. J Physiol (Lond). 1996;492(pt 2):419-430.

44. Quayle JM, McCarron JG, Brayden JE, Nelson MT. Inward rectifier $\mathrm{K}^{+}$currents in smooth muscle cells from rat resistancesized cerebral arteries. Am J Physiol. 1993;265(5 pt 1):C1363-C1370.

45. Liu YL, et al. Noxa upregulation by oncogenic activation of MEK/ERK through CREB promotes autophagy in human melanoma cells. Oncotarget. 2014;5(22):11237-11251.

46. Seok S, et al. Transcriptional regulation of autophagy by an FXR-CREB axis. Nature. 2014;516(7529):108-111.

47. Nollet M, et al. Autophagy in osteoblasts is involved in mineralization and bone homeostasis. Autophagy. 2014;10(11):19651977.

48. Klionsky DJ, et al. Guidelines for the use and interpretation of assays for monitoring autophagy in higher eukaryotes. Autophagy. 2008;4(2):151-175

49. Mackey RH, Venkitachalam L, Sutton-Tyrrell K. Calcifications, arterial stiffness and atherosclerosis. Adv Cardiol. 2007;44:234-244 
50. Gauthier-Bastien A, Ung RV, Larivière R, Mac-Way F, Lebel M, Agharazii M. Vascular remodeling and media calcification increases arterial stiffness in chronic kidney disease. Clin Exp Hypertens. 2014;36(3):173-180.

51. Molnar AO, et al. Lower serum magnesium is associated with vascular calcification in peritoneal dialysis patients: a cross sectional study. BMC Nephrol. 2017;18(1):129.

52. Kircelli F, et al. Magnesium reduces calcification in bovine vascular smooth muscle cells in a dose-dependent manner. Nephrol Dial Transplant. 2012;27(2):514-521.

53. D'Elia L, Barba G, Cappuccio FP, Strazzullo P. Potassium intake, stroke, and cardiovascular disease a meta-analysis of prospective studies. J Am Coll Cardiol. 2011;57(10):1210-1219.

54. Sica DA1, Struthers AD, Cushman WC, Wood M, Banas JS Jr, Epstein M. Importance of potassium in cardiovascular disease. J Clin Hypertens (Greenwich). 2002;4(3):198-206.

55. Diehlmann A, Bork S, Saffrich R, Veh RW, Wagner W, Derst C. KATP channels in mesenchymal stromal stem cells: strong upregulation of Kir6.2 subunits upon osteogenic differentiation. Tissue Cell. 2011;43(5):331-336.

56. Guauque-Olarte S, et al. Calcium signaling pathway genes RUNX2 and CACNA1C are associated with calcific aortic valve disease. Circ Cardiovasc Genet. 2015;8(6):812-822.

57. Motro M, Shemesh J. Calcium channel blocker nifedipine slows down progression of coronary calcification in hypertensive patients compared with diuretics. Hypertension. 2001;37(6):1410-1413.

58. Wamhoff BR, et al. L-type voltage-gated $\mathrm{Ca}^{2+}$ channels modulate expression of smooth muscle differentiation marker genes via a rho kinase/myocardin/SRF-dependent mechanism. Circ Res. 2004;95(4):406-414.

59. Freise C, Querfeld U. Inhibition of vascular calcification by block of intermediate conductance calcium-activated potassium channels with TRAM-34. Pharmacol Res. 2014;85:6-14.

60. Chen NX, Kircelli F, O'Neill KD, Chen X, Moe SM. Verapamil inhibits calcification and matrix vesicle activity of bovine vascular smooth muscle cells. Kidney Int. 2010;77(5):436-442.

61. Garat CV, et al. Platelet-derived growth factor BB induces nuclear export and proteasomal degradation of CREB via phosphatidylinositol 3-kinase/Akt signaling in pulmonary artery smooth muscle cells. Mol Cell Biol. 2006;26(13):4934-4948.

62. Zhang H, et al. Activation of PKA/CREB signaling is involved in BMP9-induced osteogenic differentiation of mesenchymal stem cells. Cell Physiol Biochem. 2015;37(2):548-562.

63. Tintut Y, Patel J, Parhami F, Demer LL. Tumor necrosis factor-alpha promotes in vitro calcification of vascular cells via the cAMP pathway. Circulation. 2000;102(21):2636-2642.

64. Ren SY, Xu X. Role of autophagy in metabolic syndrome-associated heart disease. Biochim Biophys Acta. 2015;1852(2):225-231.

65. Rajawat YS, Bossis I. Autophagy in aging and in neurodegenerative disorders. Hormones (Athens). 2008;7(1):46-61.

66. Geer JC, Mc GH Jr, Strong JP. The fine structure of human atherosclerotic lesions. Am J Pathol. 1961;38:263-287.

67. Chen K, Zhou X, Sun Z. Haplodeficiency of Klotho gene causes arterial stiffening via upregulation of scleraxis expression and induction of autophagy. Hypertension. 2015;66(5):1006-1013.

68. Gómez-Puerto MC, Verhagen LP, Braat AK, Lam EW, Coffer PJ, Lorenowicz MJ. Activation of autophagy by FOXO3 regulates redox homeostasis during osteogenic differentiation. Autophagy. 2016;12(10):1804-1816.

69. Ozeki N, et al. MicroRNA-211 and autophagy-related gene 14 signaling regulate osteoblast-like cell differentiation of human induced pluripotent stem cells. Exp Cell Res. 2017;352(1):63-74.

70. Martinet W, Schrijvers DM, Timmermans JP, Bult H. Interactions between cell death induced by statins and 7-ketocholesterol in rabbit aorta smooth muscle cells. Br J Pharmacol. 2008;154(6):1236-1246.

71. Martinet W, De Bie M, Schrijvers DM, De Meyer GR, Herman AG, Kockx MM. 7-Ketocholesterol induces protein ubiquitination, myelin figure formation, and light chain 3 processing in vascular smooth muscle cells. Arterioscler Thromb Vasc Biol. 2004;24(12):2296-2301

72. Martinet W, De Meyer GR. Autophagy in atherosclerosis: a cell survival and death phenomenon with therapeutic potential. Circ Res. 2009;104(3):304-317.

73. Yao L, Wang J, Tian BY, Xu TH, Sheng ZT. Activation of the Nrf2-ARE signaling pathway prevents hyperphosphatemiainduced vascular calcification by inducing autophagy in renal vascular smooth muscle cells [published online ahead of print May 17, 2017]. J Cell Biochem. doi: 10.1002/jcb.26137.

74. Xu M, Liu L, Song C, Chen W, Gui S. Ghrelin improves vascular autophagy in rats with vascular calcification. Life Sci. 2017;179:23-29.

75. Dai XY, et al. Phosphate-induced autophagy counteracts vascular calcification by reducing matrix vesicle release. Kidney Int. 2013;83(6):1042-1051

76. Heath JM, et al. Activation of AKT by O-linked N-acetylglucosamine induces vascular calcification in diabetes mellitus. Circ Res. 2014;114(7):1094-1102.

77. Rhoden KJ, Douglas JS. Evidence of Na-K-Cl cotransport in airway smooth muscle. Am J Physiol. 1995;268(4 pt 1):L551-L557.

78. Smith JB, Cragoe EJ Jr, Smith L. Na $/ \mathrm{Ca}^{2+}$ antiport in cultured arterial smooth muscle cells. Inhibition by magnesium and other divalent cations. J Biol Chem. 1987;262(25):11988-11994.

79. Altura BT, Zhang AM, Altura BM. Sodium-calcium exchange mechanism in vascular smooth muscle tissue as revealed by manipulating external magnesium. Magnes Trace Elem. 1990;9(3):163-175

80. Jono S, et al. Phosphate regulation of vascular smooth muscle cell calcification. Circ Res. 2000;87(7):E10-E17.

81. Witzenbichler B, Kureishi Y, Luo Z, Le Roux A, Branellec D, Walsh K. Regulation of smooth muscle cell migration and integrin expression by the Gax transcription factor. J Clin Invest. 1999;104(10):1469-1480. 